\title{
Dp2-induced autoantigen/autoantibody production from patients with systemic lupus erythematosus is not affected by AhR agonist
}

\author{
Sui-Chu Yin ${ }^{1}$, Ching-Yun Chang ${ }^{2}$ and Jaw-Ji Tsai ${ }^{3,4 *}$ \\ ${ }^{1}$ Center for Translational Medicine, Department of Medical Research, Taichung Veterans General Hospital, Taichung, Taiwan \\ ${ }^{2}$ Department of Otolaryngology, Taichung Veterans General Hospital, Taichung, Taiwan \\ ${ }^{3}$ Section of Allergy, Immunology and Rheumatology, Department of Internal Medicine, Asia University Hospital, Taichung, Taiwan \\ ${ }^{4}$ Institute of Clinical Medicine, National Yang-Ming University, Taipei, Taiwan
}

\begin{abstract}
Systemic Lupus Erythematosus (SLE) is a multifactorial systemic autoimmune disorder that can produce numerous abnormalities in cellular and humoral immune responses, including abnormal autoantigen and autoantibody production by dysregulated B cells. There are many known ligands of the AhR (aryl hydrocarbon receptor) in air pollutants, such as 6-formylindolo[3,2-b] Carbazole (FICZ) and 2,3,7,8-tetrachlorodibenzo-p-dioxin (TCDD). This study investigated the effect of FICZ/TCDD on the autoantigen/autoantibody and inflammatory cytokine production of B cells in Dp-allergic SLE. Dp2-induced autoantigen/autoantibody from $\mathrm{B}$ cell lines and PBMC derived from Dp-allergic SLE were used to evaluate the effects of FICZ/TCDD. Autoantigen/autoantibody and inflammatory cytokines were measured after cells were stimulated with FICZ/TCDD in conjuction with Dp2. The results showed that both FICZ/TCDD can activate AhR expression and induce the mRNA expression of IL- 8 . There were no effects on the production of IFN- $\alpha$, IL- 6 , or autoantigen/autoantibody, except enolase-1. In the presence of Dp2 there were no augmentation effects of FICZ/TCDD on the expression of AhR or any of the autoantigens/autoantibodies from B cell lines. Although Dp2 could induce IL-8 production from PBMC derived from Dp-allergic SLE and non-SLE patients, there were no differences between the two groups and FICZ also showed no augmentation effects on IL-8 production. In conclusion, although FICZ/TCDD can induce AhR expression and IL-8 production from B cells derived from Dp-allergic SLE. There were no augmentation effects on Dp2-induced autoantigen/autoantibody or inflammatory cytokine production by FICZ/TCDD. In the presence of Dp2, FICZ had no augmentation effect on IL-8 production from PBMC derived from patients with Dp-allergic SLE.
\end{abstract}

\section{Introduction}

Systemic Lupus Erythematosus (SLE) is a multifactorial systemic autoimmune disorder which affects multiple organs including the skin, lung, joints, kidneys, and heart [1]. The cause of SLE is unknown; however, many abnormalities in cellular and humoral immune responses including autoantibody production by dysregulated B cells, aberrant immune cell activation due to abnormal function of antigen presenting cells (APC), and autoantigen production have been reported in SLE patients $[2,3]$. Autoantigens can break self-tolerance and trigger autoimmune $\mathrm{B}$ and $\mathrm{T}$ cell responses. Disruption of the immunological tolerance to autoantigens leads to activation of autoreactive B cells and can cause increased production of autoantibodies. It has been reported that photosensitivity after sunlight exposure is a clinical feature of SLE [2], and exposure to inhaled pollutants may increase the risk of disease activity in children with juvenile-onset SLE in a large urban center [4].

The risk of developing allergy is directly related to the acquisition of immediate hypersensitivity to environmental allergens [5]. The group 2 antigen of Dermatophagoides pteronyssinus (Dp2) is one of the major Dp allergens, which has been reported to cause IgE immune responses in the majority of allergic patients. Dp2 can induce bystander activation of B cells to generate autoantigens and stimulate production of autoanitbodies in Dp-sensitive SLE patients [6].

Aryl hydrocarbon receptor, a ligand-activated transcription factor, is a sensor of exogenous and endogeneous chemicals which trigger cellular responses by regulating target genes [7]. Activation of the AhR leads to hepatocellular damage, thymic involution, cancer, and immunosuppression [8]. There are many known ligands of the AhR including 2,3,7,8-tetrachlorodibenzo-p-dioxin (TCDD) $[9,10]$ and 6-formylindolo[3,2-b] carbazole (FICZ) (11). FICZ is formed by exposure of tryptophan (Trp) to UVB light $[11,12]$. Intracellular formation of FICZ can interact with AhR resulting in translocation of AhR into the nucleus and induction of gene expression, as well as exacerbation of inflammation [13]. TCDD has been associated with numerous toxic outcomes in humans at high doses, including severe chloracne, neurotoxicity, and tumorigenesis [14]. UVB is a potential lupus activation factor and FICZ is a chemical compound formed by UVB-irradiation from tryptophan. Our previous study showed Dp2induced autoantigen/autoantibody production in patients with SLE is done through inflammasome activation [15]. It would be useful to know whether FICZ can induce autoantigen/autoantibody production and inflammatory cytokine release from $\mathrm{B}$ cells derived from patients with SLE. In this study we aimed to investigate the effects of FICZ on

${ }^{\star}$ Correspondence to: Jaw-Ji Tsai, Section of Allergy, Immunology and Rheumatology, Department of Internal Medicine, Asia University Hospital, Taichung, Taiwan, Tel. +886-4-37061688 (ext.1027); Fax: +886-4-37061673; E-mail: D00010@auh.org.tw; jawjitsai@gmail.com

Key words: SLE, AhR, FICZ, TCDD, Dp2

Received: January 28, 2019; Accepted: February 21, 2019; Published: February 26,2019 
AhR expression and autoantigen/antibody production in B cells derived from patients with Dp-sensitive SLE.

\section{Materials and methods}

\section{Selection of patients}

HDM-sensitive SLE patients (mite-specific IgE positive serum as measured by the Pharmacia CAP System, Uppsala, Sweden) were selected from the clinic of the Division of Allergy, Immunology and Rheumatology of Taichung Veterans General Hospital. Diagnosis of SLE was made according to the 1997 America College of Rheumatology revised classification criteria for SLE. This study was supported by Taichung Veterans General Hospital (TCVGH-1063804C and CE16110B).

\section{Cell cultures}

For cell separation and culture, 16-mL blood samples were collected, and the PBMCs were separated by density centrifugation using the Ficoll-Paque Plus density gradient (Pharmacia Biotech, Freiburg, Germany). Purified B cells from the PBMCs were prepared as previously described. B cell preparations were $95 \%$ positive for the CD19 marker as determined by FACS analysis. The cells were maintained in a RPMI-1640 medium containing 10\% heat inactivated FBS and $1 \%$ streptomycin/penicillin in a humidified $5 \% \mathrm{CO}_{2}$ atmosphere.

\section{Dp2 preparation}

Purified recombinant protein Dp2 (RP-DP2C-1) was purchased from Indoor Biotechnologies (Charlottesville, Virginia, USA).

\section{Chemicals}

FICZ ( $\geq 95 \%$ ) was obtained from Enzo Life Sciences (Farmingdale, NY, USA) and TCDD was purchased from Sigma-Aldrich (Stockholm, Sweden).

\section{Reverse-transcription polymerase chain reaction (RT-PCR) analysis}

Total RNA extraction was performed using a TRIZOL reagent protocol. The concentration and purity of RNA were determined by measuring the absorbance at a wavelength of $260 / 280 \mathrm{~nm}$ in a NanoDrop ND-1000 spectrophotometer (Thermo Fisher Scientific, Inc., Wilmington, DE). First-strand cDNA was synthesized by reverse transcription using a SuperScript III first-strand cDNA synthesis kit according to the manufacturer's instructions. All PCR assays were performed using PCR master mix. Primers used for PCR analyses were designed and synthesized by Clontech Laboratories (Palo Alto, CA). A suitable temperature cycle profile for each PCR reaction was determined using an MJ Mini Personal Thermal Cycler (Bio-Rad, Foster, CA). GAPDH was used as the internal control. The PCR products were loaded onto a $2 \%$ agarose gel and separated by electrophoresis. After ethidium bromide staining, DNA bands were visualized and photographed under UV-light. The intensities of the DNA bands in the agarose gel were quantified using Gel-Pro Analyzer (Media Cybernetics, Inc., MD).

\section{Enzyme-linked Immunosorbent Assay (ELISA)}

ELISA was used to determine autoantibody levels in the cell culture supernatants. The cell supernatants were collected after Dp2, FICZ, or TCDD treatment during the indicated times, and the levels of IL8 , anti-PGK-1, and anti-TRIM-21 were quantified by ELISA. Plates were read on a SpectraMax M2 microplate reader (Molecular Devices, CA, USA), and analyzed with SOFTmax analysis software (Molecular
Devices, CA, USA). The means of triplicate ELISA values for each of the dose relationships among these protein expressions were calculated by linear regression.

\section{Western blotting}

Whole cell lysates were prepared as previously described [4]. After blocking, the blots were incubated with antibodies for anti-human PGK-1, TRIM-21, Enolase-1, and $\beta$-actin, (Santa Cruz Biotechnology; Millipore, Massachusetts, USA) in TBS overnight at $4^{\circ} \mathrm{C}$ using $0.1 \%$ Tween 20 , followed by three 10 -minute washes in TBS with $0.1 \%$ Tween 20 . The membranes were then incubated with horseradish peroxidaseconjugated, secondary antibodies (Millipore, Massachusetts, USA) for one hour. Detection was performed with ECL (Millipore, Massachusetts, USA), and chemiluminescence was detected by LAS 3000. Band intensity was analyzed by Multi Gauge software V 3.0.

\section{Statistical analysis}

Statistical analyses were performed using GraphPad Prism 5 (GraphPad Software, San Diego, CA, USA). Data are presented as mean \pm Standard Error of the Mean (SEM). P-values $\leq 0.05$ were considered statistically significant. The pair Student's t test was used to determine the statistical differences between groups.

\section{Results}

\section{Effect of Dp2, FICZ, and Dp2 in conjunction with FICZ (Dp2+FICZ) on the mRNA expression in B cell lines}

$B$ cell lines derived from Dp-allergic SLE $(n=4)$ were cultured with Dp2, FICZ, Dp2+FICZ for 6 hrs. Cell pellets were collected for AhR, IFN- $\alpha$, IL6, and IL-8 measurement. The results showed AhR expression was increased by both Dp2 and FICZ (Figure 1A). When the cytokines were measured, there was significantly increased IL-8 production by both Dp2 $(1.5 \mu \mathrm{g} / \mathrm{mL})$ and FICZ (100nM and 500nM) (Figure 1A). There were no effects of FICZ on the production of IFN- $\alpha$ and IL6 , although IFN- $\alpha$ was elevated by Dp2 (Figure 1A). There were no synergistic effects of Dp2 and FICZ on the expression of AhR, IFN- $\alpha$, IL6, or IL-8 (Figure 1B).

\section{Effect of Dp2, TCDD, and Dp2 in conjunction with FICZ (Dp2+TCDD) on mRNA expression in B cells lines}

$B$ cell lines derived from Dp-allergic SLE $(n=4)$ were cultured with Dp2, TCDD, Dp2+TCDD for 6 hrs. Cell pellets were collected for AhR, IFN-a, IL6, and IL-8 measurement. The results showed AhR expression was increased by both Dp2 and TCDD. When the cytokines were measured there was significantly increased IL- 8 production by both Dp2 $(1.5 \mu \mathrm{g} / \mathrm{mL})$ and TCDD $(20 \mathrm{nM})$ (Figure 2A). There were no effects of TCDD on the production of IFN- $\alpha$ and IL-6, although IFN- $\alpha$ was elevated by Dp2 (Figure 2A). There was no synergistic effect of Dp2 and TCDD on the expression of AhR, IFN- $\alpha$, IL6, or IL-8 (Figure 2B).

\section{Effect of Dp2, FICZ, or TCDD on autoantigen production in $\mathrm{B}$ cell lines}

B cells derived from patients with Dp-allergic SLE $(n=4)$ were cultured with Dp2, FICZ, or TCDD for 5 days. Cell pellets were collected for the measurement of autoantigen. Dp2 induced autoantigen production of PGK-1, TRIM-21, and Enolase-1 in B cells (Figure 3A). There was significantly increased Enolase production by Dp2, FICZ, and TCDD (Figure 3A). There were no synergistic effects of Dp2, FICZ, or TCDD on the expression of PGK-1, TRIM-21, and Enolase-1 (Figure 3B). 


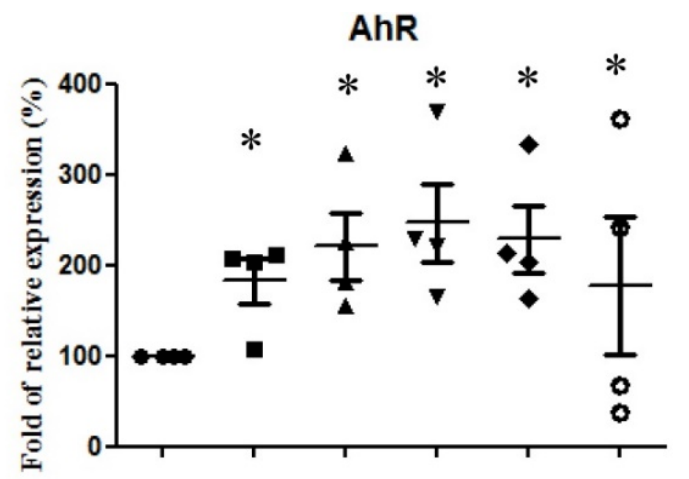

IFN- $\alpha$

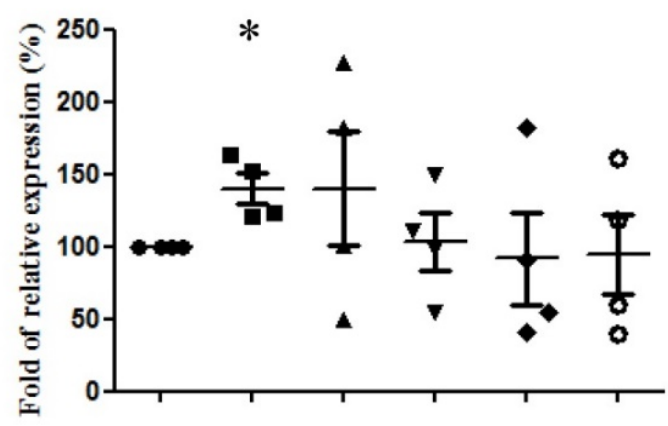

IL-6

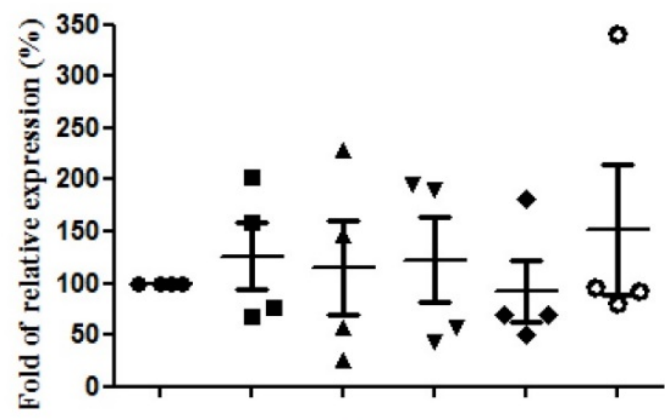

IL-8

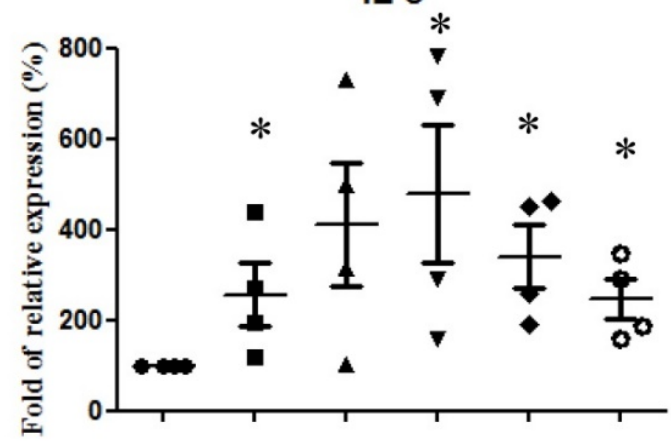

$\operatorname{LPS}(\mu \mathrm{g} / \mathrm{mL}) \quad-\quad-\quad-\quad-\quad-\quad-\quad-0.5$ Dp2 $(\mu \mathrm{g} / \mathrm{mL}) \quad-\quad 1.5 \quad-\quad-7 \quad-\quad-$

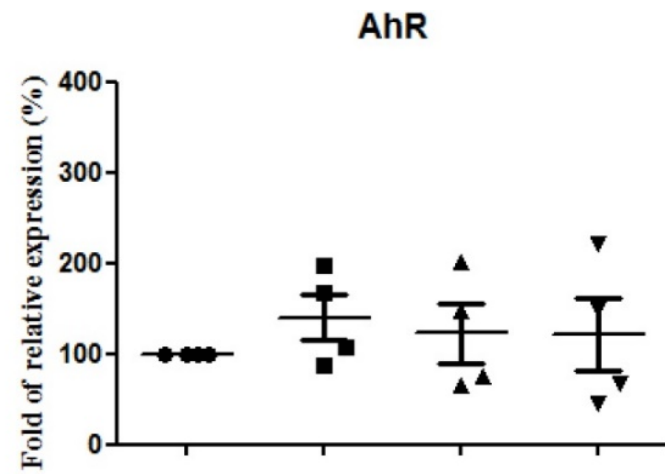

IFN- $\alpha$

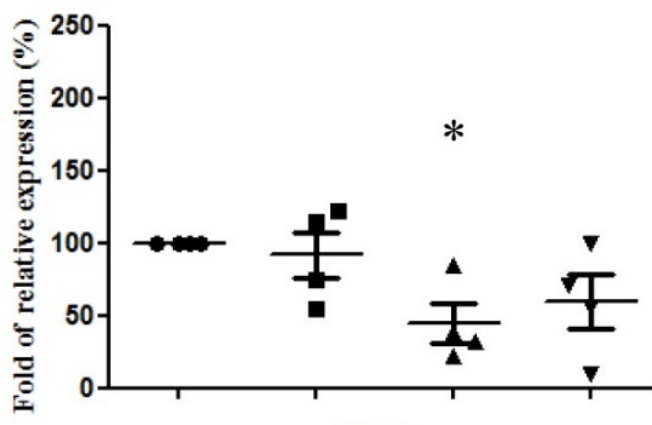

IL-6

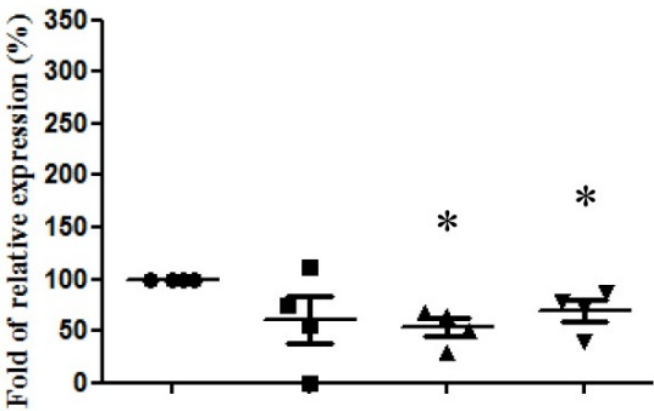

IL-8

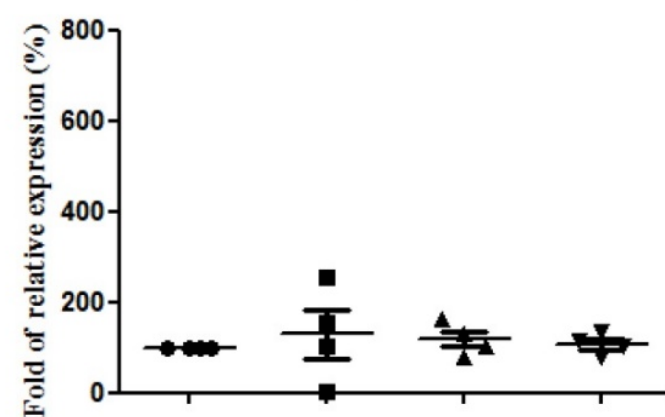

LPS $(\mu \mathrm{g} / \mathrm{mL})$ Dp2 $(\mu \mathrm{g} / \mathrm{mL})$ FICZ (nM)

Figure 1. Effect of Dp2, FICZ and Dp2 in conjunction with FICZ (Dp2+FICZ) on the mRNA expression from B cell lines: B cells derived from patients with Dp-allergic SLE (n=4) were incubated with $\mathrm{Dp} 2$ for 6 hours in a $\mathrm{CO}_{2}$ incubator. Cell pellets were collected for the AhR, IFN- $\alpha$, IL- 6 , and IL- 8 measurements. * $p<0.05$ in comparison with buffer control (A). $* p<0.05$ in comparison with $\mathrm{Dp} 2(\mathrm{~B})$ 


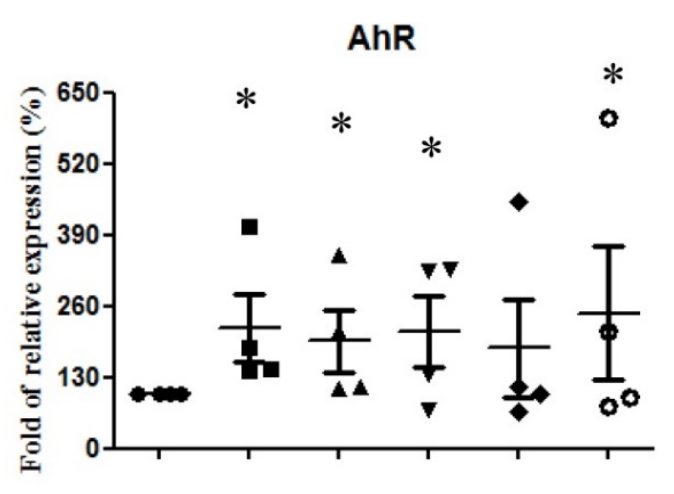

IFN- $\alpha$
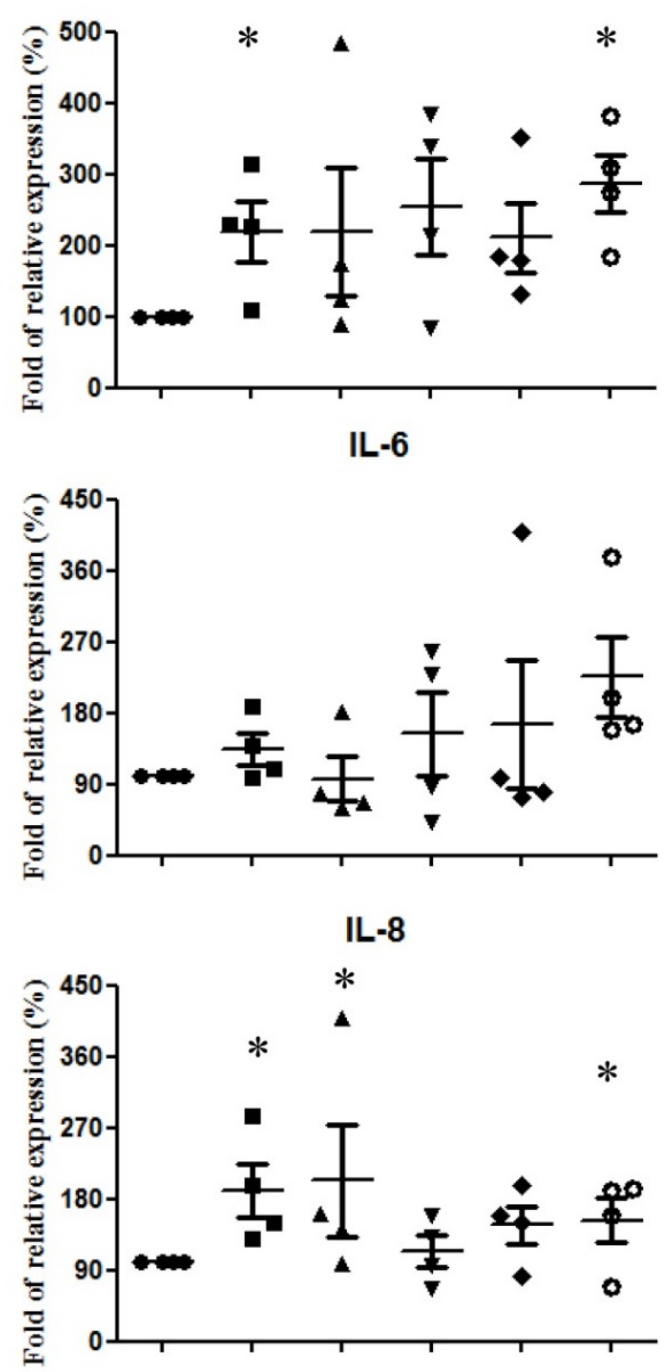

$\operatorname{LPS}(\mu \mathrm{g} / \mathrm{mL}) \quad-\quad-\quad-\quad-\quad-\quad-0.5$

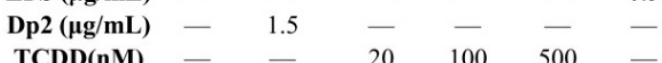

AhR

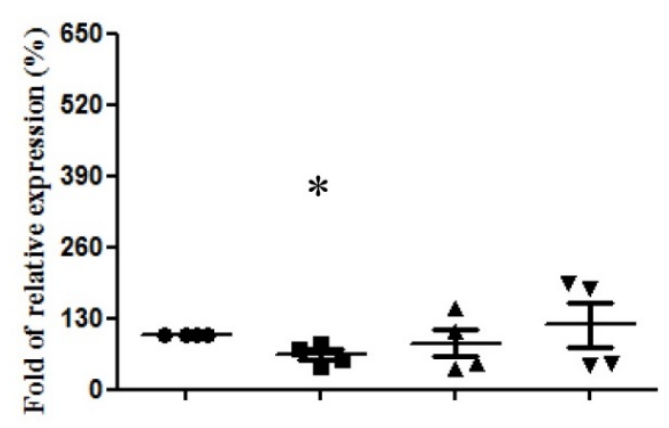

IFN- $\alpha$
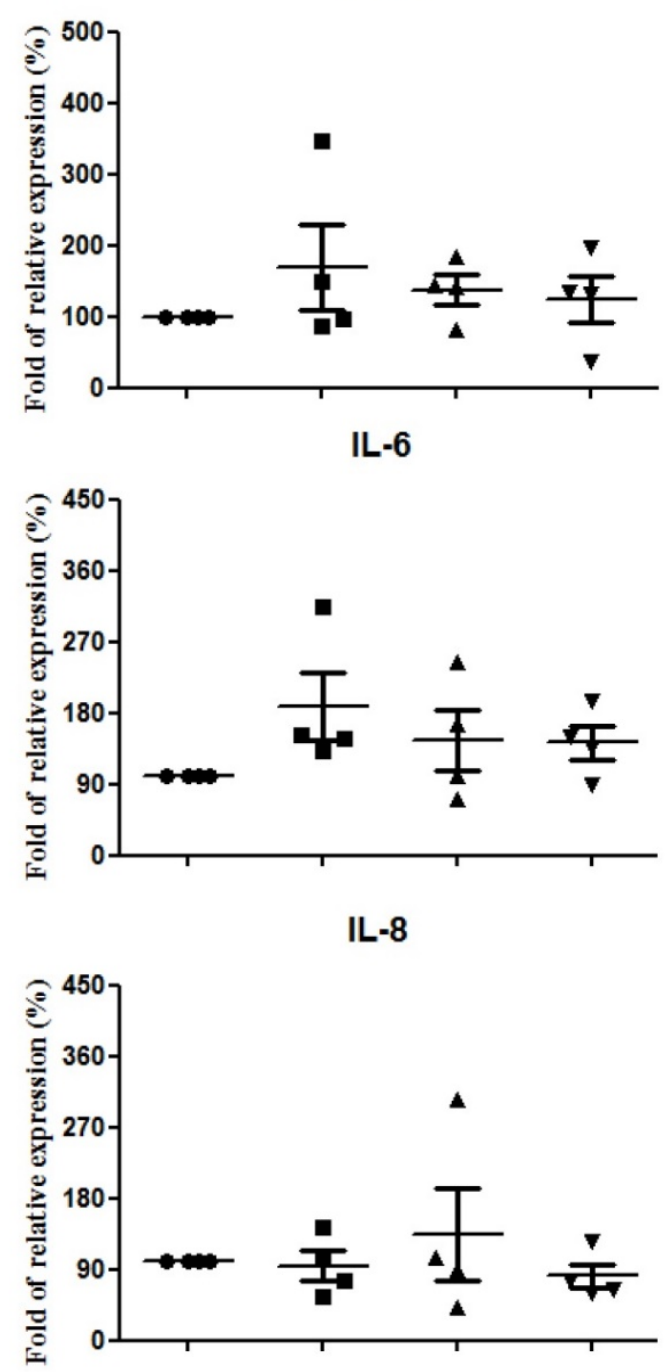

LPS $(\mu \mathrm{g} / \mathrm{mL})$ $\mathrm{Dp} 2(\mu \mathrm{g} / \mathrm{mL})$ TCDD (nM)

Figure 2. Effect of Dp2, TCDD, and Dp2 in conjunction with TCDD (Dp2+TCDD) on the mRNA expression in B cell lines: B cells derived from patients with Dp-allergic SLE ( $\mathrm{n}=4$ ) were incubated with $\mathrm{Dp} 2$ for 6 hours in a $\mathrm{CO}_{2}$ incubator. Cell pellets were collected for AhR, IFN- $\alpha$, IL-6, and IL-8 measurements. ${ }^{*} p<0.05$ in comparison with buffer control (A). * $p<0.05$ in comparison with Dp2 (B) 
PGK-1

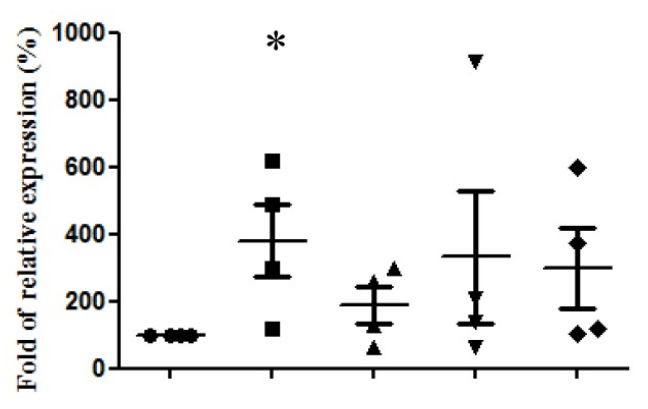

TRIM-21

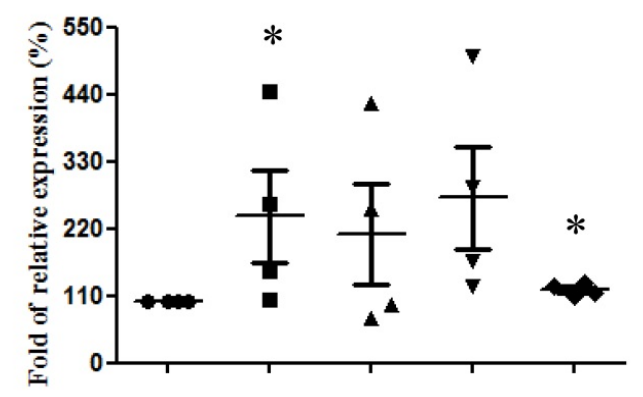

Enolase-1

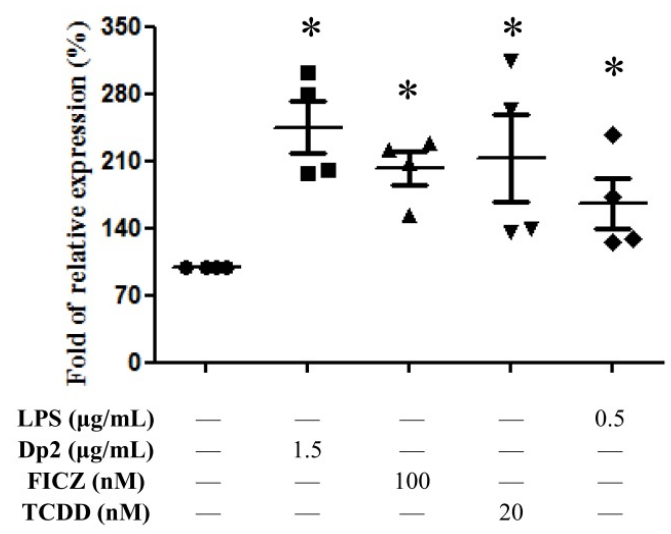

PGK-1

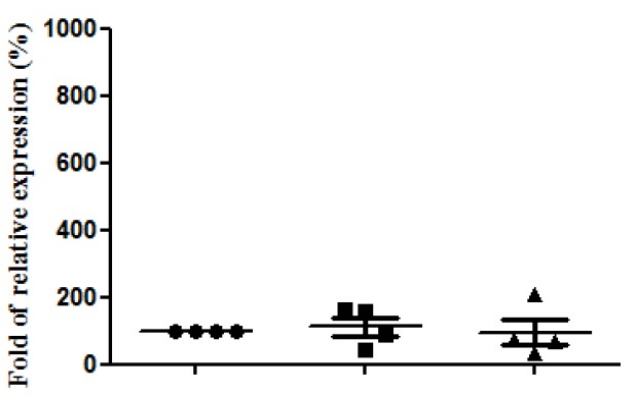

TRIM-21

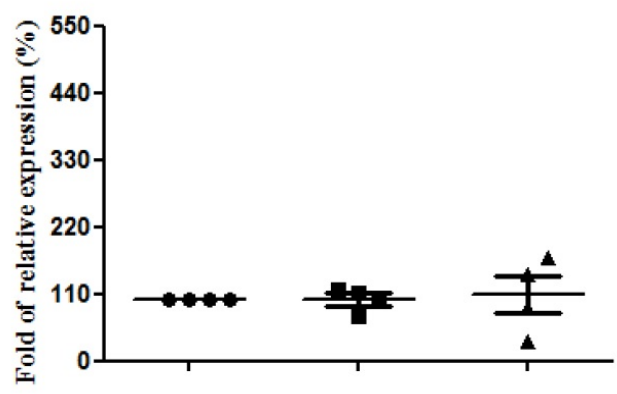

Enolase-1

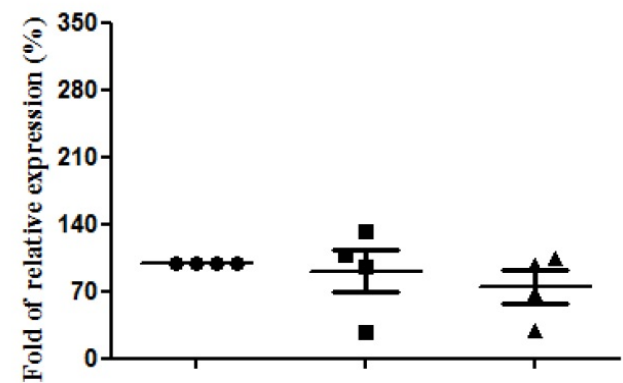

$\begin{array}{cccc}\text { LPS }(\boldsymbol{\mu g} / \mathbf{m L}) & - & - & - \\ \text { Dp2 }(\boldsymbol{\mu g} / \mathbf{m L}) & 1.5 & 1.5 & 1.5 \\ \text { FICZ }(\mathbf{n M}) & - & 100 & - \\ \text { TCDD }(\mathbf{n M}) & - & - & 20\end{array}$

Figure 3. Effect of Dp2, FICZ, or TCDD on autoantigen production in B cell lines: B cells $(n=4)$ derived from patients with Dp-allergic SLE were treated with Dp2, FICZ, or TCDD for 5 days in a $\mathrm{CO}_{2}$ incubator. Cell pellets were collected for the measurement of autoantigens. ${ }^{*} p<0.05$ in comparison with buffer control (A). ${ }^{*} p<0.05$ in comparison with Dp2 (B)

Effect of Dp2, FICZ, or TCDD on autoantibody production in $\mathrm{B}$ cell lines

$B$ cells derived from patients with Dp-allergic SLE were incubated with Dp2 for 5 days. Cell cultured supernatant was collected for the measurement of autoantibodies. The results showed FICZ and TCDD had no effects on anti-PGK-1 and anti-TRIM-21 production, although the production of anti-PGK-1 and anti-TRIM21 could be upregulated by Dp2 and LPS (Figure 4). There were no synergistic effects of Dp2, FICZ, or TCDD on the expression of anti-PGK-1 and anti-TRIM21 (Figure 4).

\section{Effect of Dp2 and Dp2 in conjunction with FICZ (Dp2+FICZ) on IL-8 secretion by PBMCs}

PBMCs derived from Dp-allergic SLE and Dp-allergic nonSLE were cultured with Dp2 and Dp2+FICZ for 3 days. Cell culture supernatant was collected for the measurement of IL-8. Results showed
Dp2 could enhance IL-8 production in both groups of patients. These were no significant difference between these two groups. In the cell supernatant of Dp2+FICZ the secretion of IL-8 was not enhanced in either group of patients (Figure 5A). When the production of IL-8 was compared between Dp-allergic SLE and Dp-allergic non-SLE, there were also no significant differences between the Dp2 and Dp2+FICZ groups (Figure 5B).

\section{Discussion}

The effects of AhR agonist FICZ/TCDD on the production of autoantigen/autoantibody and production of inflammatory cytokines in patients with Dp-allergenic SLE were investigated. Our results showed FICZ/TCDD could induce AhR and IL-8 expression at concentrations of 100 and $500 \mu \mathrm{M}$ of FICZ and $20 \mu \mathrm{M}$ of TCDD; however, their expression could be abolished by Dp2 in conjunction with FICZ/ TCDD. The expressions of IFN- $\alpha$ and IL- 6 were significantly reduced by FICZ, but not by TCDD when co-cultured with Dp2. 
Anti-PGK-1

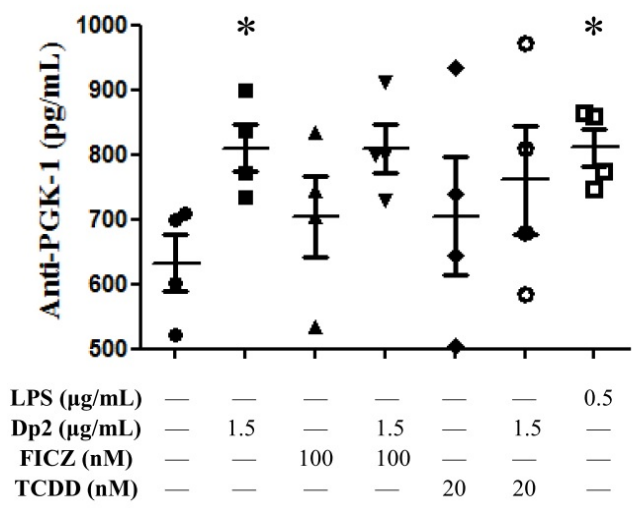

Anti-TRIM-21

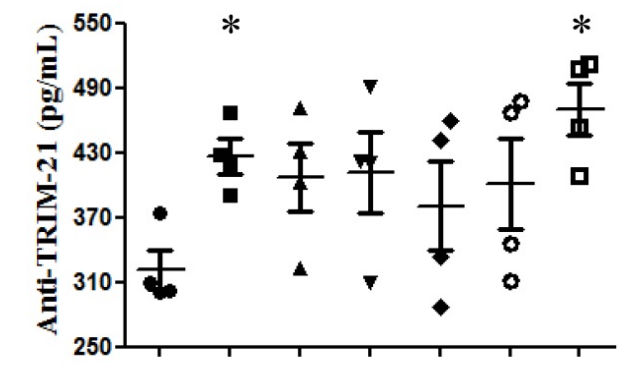

$\begin{array}{cccccccc}\text { LPS }(\boldsymbol{\mu g} / \mathbf{m L}) & - & - & - & - & - & - & 0.5 \\ \operatorname{Dp2}(\boldsymbol{\mu g} / \mathbf{m L}) & - & 1.5 & - & 1.5 & - & 1.5 & - \\ \text { FICZ }(\mathbf{n M}) & - & - & 100 & 100 & - & - & - \\ \operatorname{TCDD}(\mathbf{n M}) & - & - & - & - & 20 & 20 & -\end{array}$

Figure 4. Effect of Dp2, FICZ, or TCDD on autoantibody production in B cell lines: B cells ( $\mathrm{n}=4$ ) derived from Dp-allergic SLE were incubated with Dp2, FICZ, or TCDD for 5 days in a $\mathrm{CO}_{2}$ incubator. Cell culture supernatant was collected for the measurement of autoantibodies. ${ }^{*} p<0.05$ in comparison with buffer control

IL-8

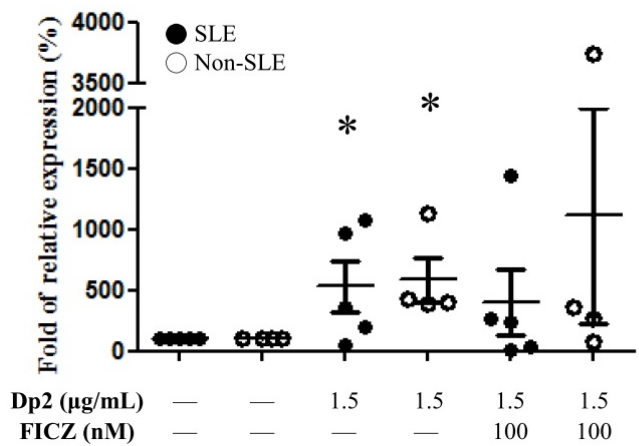

IL-8

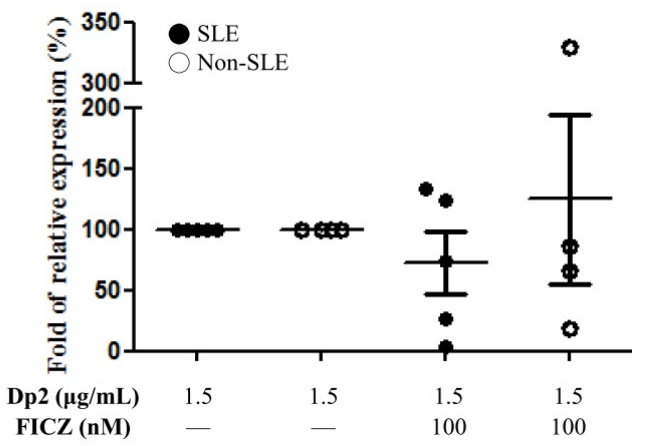

Figure 5. Effect of Dp2, FICZ, and Dp2 in conjunction with FICZ (Dp2+FICZ) on IL-8 expression from PBMCs: PBMCs derived from Dp-allergic SLE ( $\mathrm{n}=5$ ) and Dp-allergic non-SLE $(\mathrm{n}=4)$ were incubated with $\mathrm{Dp} 2$ and/or FICZ for 3 days in the $\mathrm{CO}_{2}$ incubator. Cell culture supernatant was collected for the measurement of IL-8. * $p<0.05$ in comparison with buffer control (A). No differences in comparison with Dp2 (B)

This discrepancy between FICZ and TCDD could be due to the different functions and affinity to AhR involved in the cell activation. It has been reported that FICZ can be efficiently degraded by the prototypical AhR target CYP1A1, leading to negative feedback loops and transient AhR activation. In contrast, TCDD can sustain AhR activation $[16,17]$. Activation of AhR by TCDD leads to the differentiation of naïve CD4+ T cells into FoxP3+ regulatory T cells (Tregs) [18]. Moreover, AhR activation via FICZ enhanced the differentiation of naive CD4+ T cells into Th17 cells [19]. Activation of AhR in vivo by TCDD reduced the severity of disease in the experimental autoimmune encephalitis (EAE) model of multiple sclerosis, while treatment with FICZ increased the severity of disease [20].

FICZ and TCDD can induce enolase-1 expression; however, its expression can be abolished by Dp2 in conjunction with either FICZ or TCDD. These results suggest that the signaling of enolase- 1 expression could be down-regulated by Dp2. Since FICZ/TCDD has no effect on the expression of PGK-1/TRIM 21 expression, their antibodies (antiPGK-1 and anti-TRIM 21) were not affected by FICZ/TCDD in the presence or absence of $\mathrm{Dp} 2$.

In B cell lines, FICZ-induced AhR and IL-8 expression could be abolished by co-culture with Dp2, and Dp2-induced IL- 8 production could be abolished by FICZ in PBMCs. These results suggest that FICZ-induced AhR and IL-8 production may be counteracted by Dp2. Our previous reports showed Dp2-induced autoantigen / autoantibody production in patients with SLE could be down-regulated by CPPecp through NLRP3 in-activation (21). It has been reported that FICZ-enhanced expression of AhR can negatively regulate NLRP3 inflammasome activation by inhibiting NLRP3 transcription [22]. Thus, it is likely Dp2-induced inflammasome activation is downregulated by FICZ.

Through AhR activation, it was possible to control B cell differentiation from the hematopoietic stem cell stage through to the pro-B cell, mature $\mathrm{B}$ cell, and autoantibody-secreting plasm cell stages [23]. In this study, FICZ/TCDD and Dp2 could activate AhR through different ligand-activated transcription factors and cytokine production. It is likely that FICZ/TCDD and Dp2 could skew the immune cell polarization and modulate the disease activity in Dpallergic SLE through different receptors and transcription factors of cytokines. A limitation of this study was the small number of Dpallergic SLE patients that were included in the analysis. Further studies are needed to elucidate the precise mechanism of interaction between FICZ/TCDD and Dp2.

\section{Conclusion}

In conclusion, we demonstrated that Dp2 could activate B cells to enhance expression of AhR and cytokines (IL-8/IFN- $\alpha$ ), as well as production of autoantigens / autoantibodies. FICZ/TCDD could also activate B cells to enhance AhR and IL- 8 expression. However, they had no augmentation effect on the production of these autoantigens/ autoantibodies in the presence of Dp2. Similarly, in the presence of Dp2, FICZ had no augmentation effect on the IL-8 production in PBMCs derived from patients with Dp-allergic SLE. 


\section{Acknowledgments}

This study was supported by a grant (TCVGH-1063804C) from Taichung Veterans General Hospital. The authors sincerely appreciate the assistance of the Center for Translational Medicine of Taichung Veterans General Hospital, Taichung, Taiwan.

\section{Conflicts of interest}

The authors have no conflicts of interest with respect to the funding presented in this manuscript.

\section{References}

1. Rahman A, Isenberg DA (2008) Systemic lupus erythematosus. N Engl J Med 358: 929-939. [Crossref]

2. Steinberg AD (1992) Concepts of pathogenesis of systemic lupus erythematosus. Clin Immunol Immunopathol 63: 19-22. [Crossref]

3. Wozniacka A, Lesiak J, Narbutt DP, McCauliffe A, Sysa-Jedrzejowska A (2006) Chloroquine treatment influences proinflammatory cytokine levels in systemic lupus erythematosus patients. Lupus, 15: 268-275. [Crossref]

4. Fernandes EC, Silva CA, Braga AL, Sallum AM, Campos LM, et al. (2015) Exposure to air pollutants and disease activity in juvenile-onset systemic lupus erythematosus patients. Arthritis Care Res (Hoboken) 67: 1609-1614. [Crossref]

5. Haydon RC $3^{\text {rd }}$ (2003) Addressing the prevalence of respiratory allergy in the home environment, Otolaryngol clin 36: 803-824.

6. Yu SJ, Liao EC, Tsai JJ (2014) Der p 2 can induce bystander activation of B cells derived from patients with systemic lupus erythematosus. Immunobiology 219: 958963. [Crossref]

7. Denison MS, Soshilov AA, He G, DeGroot DE, Zhao B (2011) Exactly the same but different: Promiscuity and diversity in the molecular mechanisms of action of the aryl hydrocarbon (dioxin) receptor. Toxicol Sci 124: 1-22. [Crossref]

8. Schmidt JV, Carver LA, Bradfield CA (1993) Molecular characterization of the murine ahr gene. Organization, promoter analysis, and chromosomal assignment. $J$ Biol Chem 268: 22203-22209. [Crossref]

9. Liu F, Liao C, Fu J, Lv J, Xue Q, et al. (2014) Polycyclic aromatic hydrocarbons and organochlorine pesticides in rice hull from a typical e-waste recycling area in southeast China: temporal trend, source, and exposure assessment. Environ Geochem Health 36: 65-77. [Crossref]
10. Lee J, Prokopec SD, Watson JD, Sun RX, Pohjanvirta R, et al. (2015) Male and female mice show significant differences in hepatic transcriptomic response to 2,3,7,8-tetrachlorodibenzo-p-dioxin. BMC Genomics 16: 625. [Crossref]

11. Fritsche E, Schäfer C, Calles C, Bernsmann T, Bernshausen T, et al. (2007) Lightening up the uv response by identification of the arylhydrocarbon receptor as a cytoplasmatic target for ultraviolet b radiation. Proc Natl Acad Sci U S A 104: 8851-8856.

12. Rannug U, Rannug A, Sjöberg U, Li H, Westerholm R, et al. (1995) Structure elucidation of two tryptophan-derived, high affinity ah receptor ligands. Chem Biol 2: 841-845. [Crossref]

13. Stockinger B, Di Meglio P, Gialitakis M, Duarte JH (2014) The aryl hydrocarbon receptor: Multitasking in the immune system. Annu Rev Immunol 32: 403-432. [Crossref]

14. Hanieh H (2004) Toward understanding the role of aryl hydrocarbon receptor in the immune system: Current progress and future trends. BioMed research international 8: 520-763.

15. Tsai JJ (2017) Dp2-induced autoantigen/autoantibody production from patients with systemic lupus erythematosus is done through AIM2 and not NLRP3. J Rheumatol Arthritic Dis 2: 1-6.

16. Smirnova A, Wincent E, Vikström Bergander L, Alsberg T, Bergman J, et al. (2016) Evidence for new light-independent pathways for generation of the endogenous aryl hydrocarbon receptor agonist FICZ. Chem Res Toxicol 29: 75-86. [Crossref]

17. Yeager RL, Reisman SA, Aleksunes LM, Klaassen CD (2009) Introducing the "TCDDinducible AhR-Nrf2 gene battery". Toxicol Sci 111: 238-246. [Crossref]

18. Quintana FJ, Basso AS, Iglesias AH, Korn T, Farez MF, et al. (2008) Control of T(reg) and $\mathrm{T}(\mathrm{H}) 17$ cell differentiation by the aryl hydrocarbon receptor. Nature 453: 65-71. [Crossref]

19. Veldhoen M, Hirota K, Westendorf AM, Buer J, Dumoutier L, et al. (2008) The ary hydrocarbon receptor links $\mathrm{t}(\mathrm{h}) 17$-cell-mediated autoimmunity to environmental toxins. Nature 453: 106-109. [Crossref]

20. Stockinger B, Di Meglio P, Gialitakis M, Duarte JH (2014) The aryl hydrocarbon receptor: Multitasking in the immune system. Annu Rev Immunol 32: 403-432. [Crossref]

21. Ye CX, Yu SJ, Chang CY, Tsai JJ (2017) Dp2-Induce autoantigen/autoantibody production from patients with systemic lupus erythematosus and its modulation by Cppecp. J Rheumatol Arthritic Dis 2: 1-6.

22. Huai W, Zhao R, Song H, Zhao J, Zhang L, et al. (2014) Aryl hydrocarbon receptor negatively regulates NLRP3 inflammasome activity by inhibiting NLRP3 transcription. Nat Commun 20: 4738. [Crossref]

23. Sherr DH, Monti S (2013) The role of the aryl hydrocarbon receptor in normal and malignant B cell development. Semin Immunopathol 35: 705-716. [Crossref]

Copyright: (C2019 Yin S. This is an open-access article distributed under the terms of the Creative Commons Attribution License, which permits unrestricted use, distribution, and reproduction in any medium, provided the original author and source are credited. 